\title{
Avaliação Sistematizada da Dificuldade de Exposição das Pregas Vocais na Microcirurgia da Laringe
}

\author{
Difficulty Systematized Evaluation of Vocal Folds Exposure in \\ Microsurgery of the Larynx
}

\author{
Annelyse Cristine Ballin*, Evaldo Dacheux de Macedo Filbo **, Gustavo B. Sela***, Guilberme S. Catani****, \\ Jorge Massaaki Ido Filbo****, Carlos Henrique Ballin*****, Marcos Mocellin******.
}

\author{
* Residente do terceiro ano do Serviço de Otorrinolaringologia do HC-UFPR \\ ** Doutor. Médico Responsável pelo Setor de Laringologia da Disciplina de Otorrinolaringologia do HC-UFPR e IPO. \\ **** Acadêmico do curso de Medicina da UFPR. \\ ***** Professor Colaborador do Setor de Laringologia do HC-UFPR. \\ ******* Doutorando do curso de Medicina da UFPR. \\ ******** Chefe e Professor Titular do Serviço de Otorrinolaringologia do HC-UFPR.
}

Instituição: Serviço de Otorrinolaringologia do Hospital de Clínicas da Universidade Federal do Paraná e Instituto Paranaense de Otorrinolaringologia Trabalho aprovado pelo Comitê de Ética dos dois Serviços.

Curitiba / PR - Brasil.

Endereço para correspondênica: Annelyse Cristine Ballin - Rua Antonio Escorsin, 674, Condomínio Don Carlo, casa 31 - Curitiba / PR - Brasil - CEP: 82015-000 - Telefone: (+55 41) 8406-8293 - Email: anneballin@uol.com.br

Artigo recebido em 11 de Fevereiro de 2010. Artigo aprovado em 31 de Maio de 2010.

Trabalho premiado no Serviço de Otorrinolaringologia do HC-UFPR por melhor trabalho realizado pelos residentes no ano de 2009

\section{RESUMO}

Introdução:

Diversos estudos abordam fatores pré-operatórios que predizem dificuldade de intubação endotraqueal, graduada pelos anestesistas pela escala de Cormack-Lehane. Estes parâmetros foram pouco avaliados para a dificuldade de locação do laringoscópio nas microcirurgias da laringe. Não há uma escala padrão de dificuldade direcionada aos cirurgiões de laringe.

Objetivo: $\quad$ Criar uma escala padrão de dificuldade de locação do laringoscópio durante microcirurgia da laringe, com foco na exposição das pregas vocais (PPVV); avaliar quais parâmetros clínicos predizem dificuldade de exposição das PPVV; verificar a melhora da exposição laríngea com o suspensor do laringoscópio.

Método: $\quad$ Estudo prospectivo, randomizado, de 57 pacientes submetidos à microcirurgia de laringe. No pré-operatório foram avaliados: 3 dados epidemiológicos, 2 de anamnese e 13 de exame físico. No intra-operatório: o anestesista avaliava o escore de Cormack-Lehane e o cirurgião avaliava conforme a escala proposta, antes e após a colocação do suspensor.

Resultados e Conclusão:Vários parâmetros apresentaram sensibilidade, especificidade, valor preditivo positivo altos para exposição inadequada da laringe. Porém, apenas distância hiomentual $<6,05 \mathrm{~cm}(\mathrm{p}=0,003)$ e classe 2 de Cormack-Lehane $(\mathrm{p}=0,04)$ com significância estatística e alta sensibilidade, $100 \%$ e $81 \%$ respectivamente. O uso do suspensor do laringoscópio melhorou a exposição laríngea de forma significativa $(\mathrm{p}=0,04)$. A escala proposta padroniza a vizualização e gradua a dificuldade de exposição das PPVV, facilitando comparações entre estudos e comunicação entre otorrinolaringologistas.

Palavras-chave: laringe, intubação, laringoscópios, microcirurgia, pregas vocais, repertório: seção laringe e traqueia.

\section{SUMMARY}

Introduction:

Several studies addressing preoperative factors that predict difficulty of endotracheal intubation graduated by anesthesiologists, for the scale of the Cormack-Lehane. These parameters were evaluated for the difficulty of location of the laryngoscope in microsurgery of the larynx. There is not a standard scale of difficulty targeted to surgeons of the larynx.

Objective: Create a standard scale of difficulty leasing the laryngoscope during microsurgery of the larynx, with a focus on exposure of the vocal folds (vocal cords) to evaluate which clinical parameters predict difficulty of exposure of their vocal folds and verify the improvement of laryngeal exposure with the hanger of the laryngoscope.

Method: A prospective randomized study, 57 patients undergoing laryngeal microsurgery. The preoperative parameters were evaluated: three epidemiological data, two of history and 13 physical examination. Intraoperatively: the anesthesiologist evaluated the Cormack-Lehane score and the surgeon evaluated according to the proposed scale, before and after placement of the hanger.

Results and Conclusion: Several parameters showed sensitivity, specificity, positive predictive value for high inadequate exposure of the larynx. But only distance hiomentual $<6.05 \mathrm{~cm}(\mathrm{p}=0.003)$ and 2 classes of Cormack-Lehane $(\mathrm{p}=0.04)$ with statistical significance and high sensitivity of $100 \%$ and $81 \%$ respectively. The use of the hanger of laryngoscope laryngeal exposure improved significantly $(\mathrm{p}=0.04)$. The proposed scale standardizes the visualization and grades the difficulty of exposure of their vocal folds, facilitating comparisons between studies and communication between otolaryngologists.

Keywords: larynx, intubation, laryngoscopes, microsurgery, vocal fold, repertory: section larynx and trachea. 


\section{INTRODUÇÃO}

A microlaringoscopia de suspensão vem sendo usada com finalidade diagnóstica e terapêutica em diversas afecções laríngeas. O ideal é a visualização de toda a prega vocal, até a comissura anterior, visando evitar erros diagnósticos, remoção incompleta de lesões, injúria inadvertida das pregas vocais (PPVV) ou até aborto do procedimento. Na maioria dos casos, o laringoscópio rígido de suspensão permite uma exposição adequada da laringe. Entretanto, alguns pacientes apresentam dificuldade de exposição laríngea, um dos principais problemas na microcirurgia da laringe.

Diversos estudos abordam fatores clínicos pré-operatórios que predizem a dificuldade de intubação endotraqueal para procedimentos cirúrgicos. São exemplos o índice de massa corporal, o índice de Mallampati modificado, a circunferência cervical, a protrusão da mandíbula, o teste da mordida do lábio superior, a distância inter-incisivos, a distância hiomentual, a distância tireomentual, a distância esternomentual, a maior distância horizontal da mandỉbula, entre outros $(1,2)$. Os anestesistas utilizam à escala de Cormack e Lehane, para graduar a dificuldade de intubação endotraqueal, com foco na visualização da laringe, através da elevação da epiglote pelo laringoscópio (1). Entretanto, estes mesmos parâmetros foram pouco avaliados para a locação do laringoscópio, na realização das microcirurgias da laringe. Além disso, não existe uma escala padrão utilizada pelos cirurgiões de laringe.

Este trabalho tem como objetivos:

1) criar uma escala padrão de dificuldade de locação do laringoscópio durante a microcirurgia da laringe, com foco na exposição das pregas vocais,

2) avaliar quais parâmetros clínicos predizem dificuldade de visualização da laringe,

3) verificar a melhora da exposição das PPVV com o suspensor do laringoscópio.

\section{MÉTODO}

Estudo prospectivo de pacientes submetidos à microcirurgia de laringe, no período de julho a dezembro de 2009, no Serviço de Otorrinolaringologia do Hospital de Clínicas de Curitiba / Universidade Federal do Paraná e Instituto Paranaense de Otorrinolaringologia.

Este trabalho foi aprovado pelo Comitê de Ética das duas Instituições.

Independente da indicação cirúrgica, todos os pacientes deste período, que concordaram em participar da pesquisa, foram submetidos a um protocolo dividido em avaliação pré-operatória e avaliação intra-operatória.

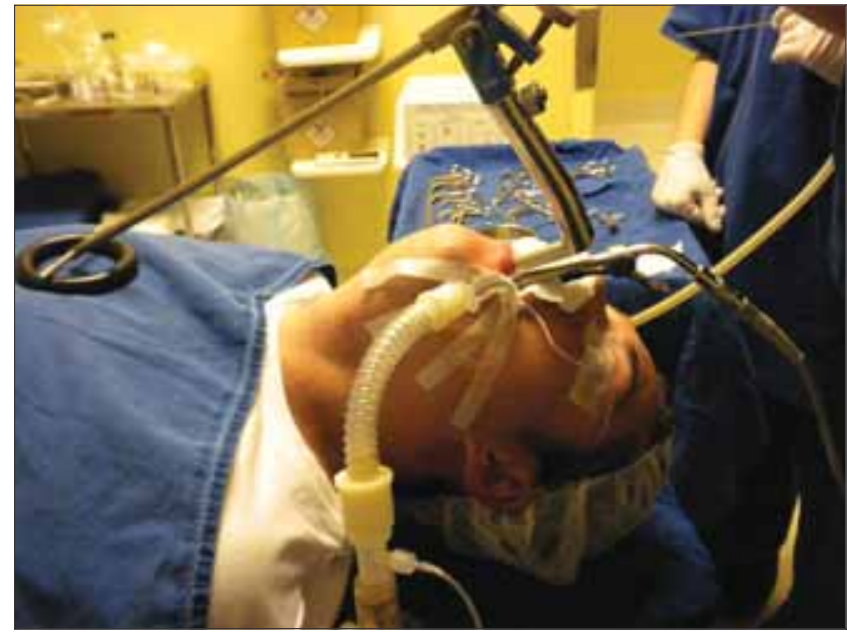

Figura 1. Posição do paciente, laringoscópio de suspenção e suspensor do laringoscópio. - Paciente pronto para realização da microcirurgia da laringe, na posição de BoyceJackson, com laringoscópio de suspenção universal de Dan e suspensor do laringoscópio alocados.

Os pacientes com idade inferior a 12 anos foram excluídos do trabalho, devido a diferenças anatômicas e pela falta de valores de referências dos índices avaliados. Também foram excluídos pacientes com doenças reumatológicas, com artrose da coluna cervical e/ou com lesões laríngeas extensas. Estes, porque essas alterações dificultavam a correta classificação das escalas de CormackLehane e da escala proposta pelos autores.

Os pacientes foram intubados com tubo endotraqueal (diâmetro de 5,5 ou 6,0 mm), sob anestesia geral e relaxante muscular. Após a intubação endotraqueal, com o paciente em decúbito horizontal, sem travesseiros sob a cabeça, pescoço ou tórax, com flexão cervical e extensão da cabeça (posição de Boyce-Jackson) (3) (Figura 1), alocava-se o laringoscópio de suspensão universal de Dan (Figuras 2 e 3). O laringoscópio universal de Dan apresenta tamanho único, $18 \mathrm{~cm}$ de comprimento, $2 \mathrm{~cm}$ de diâmetro na altura e $1,5 \mathrm{~cm}$ de diâmetro na largura. Nos casos de difícil exposição da laringe, foi utilizada compressão da pele no local da projeção da comissura anterior na cartilagem tireoide, na parte externa do pescoço. Esta, feita através de compressão manual, efetuada pela instrumentadora, ou por uma fita de esparadrapo, comprimindoa, sendo fixada nas bordas da cabeceira da mesa.

\section{Avaliação pré-operatória}

No pré-operatório, somente dois médicos intercalavam-se e coletavam os seguintes dados:

- dados epidemiológicos: idade, sexo e indicação cirúrgica;

- dados da anamnese: roncos noturnos, doença reumatológica, alteração cervical e história de trauma cervical; 


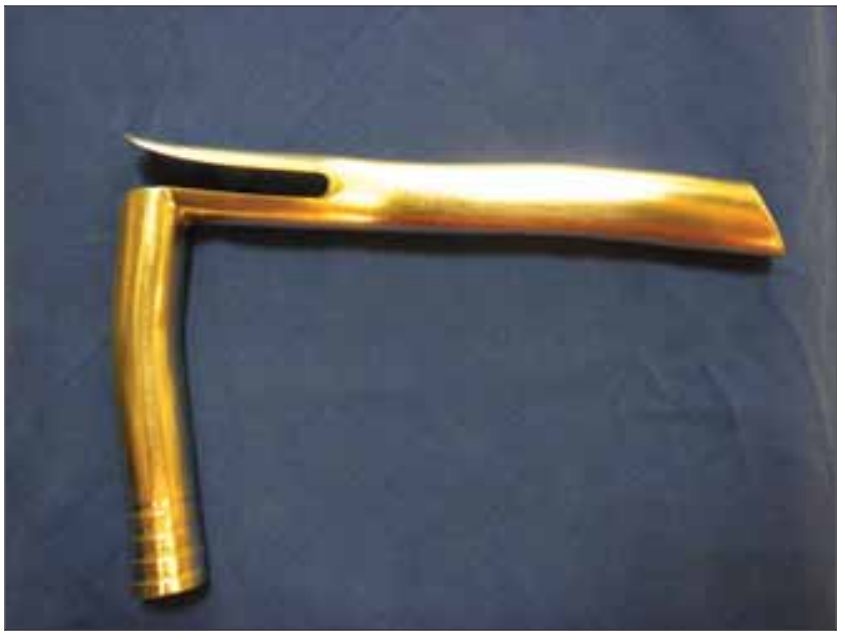

Figura 2. Laringoscópio de suspensão universal de Dan. Vista lateral.

- variáveis clínicas: peso, altura, ausência de dentes, circunferência cervical, índice de Mallampati modificado, protrusão da mandíbula (avaliada pelo teste da mordida do lábio superior), flexão e extensão do pescoço, abertura oral (avaliada pelo gap inter-incisivos), distância hiomentual na posição neutra (DHMn) e em extensão do pescoço (DHM), distância tireomentual em extensão do pescoço (DTM), distância esternomentual em extensão do pescoço (DEM) e maior distância horizontal da mandỉbula (MDHM). Foram calculados o IMC e a relação da distância hiomentual em extensão do pescoço/ distância hiomentual na posição neutra.

A circunferência cervical foi medida em centímetros na altura da cartilagem tireoide.

O índice de Mallampati modificado foi graduado da seguinte maneira: grau $1=$ tonsilas, pilares e palato mole visíveis; grau $2=$ somente úvula, pilares e pólo superior das tonsilas visíveis; grau 3 = palato mole parcialmente visível; grau $4=$ somente palato duro visível.

O teste da mordida do lábio superior foi realizado de acordo com os seguintes critérios: classe $1=$ incisivos inferiores mordem o lábio superior acima da linha do vermelhão; classe $2=$ incisivos inferiores mordem o lábio inferior abaixo da linha do vermelhão; classe $3=$ incisivos inferiores não podem morder o lábio superior (2).

A flexão da cabeça foi avaliada pedindo para o paciente encostar o pescoço no tórax. Classificada como normal quando possível, caso contrário, como limitada. Pedindo para paciente estender o pescoço, avaliava-se a extensão cervical.

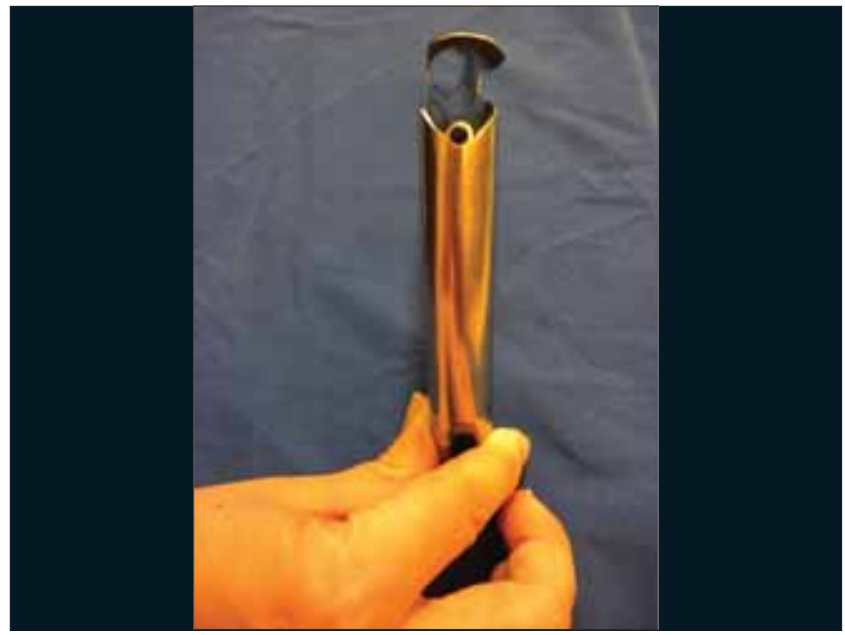

Figura 3. Laringoscópio de suspensão universal de Dan. Vista basal.

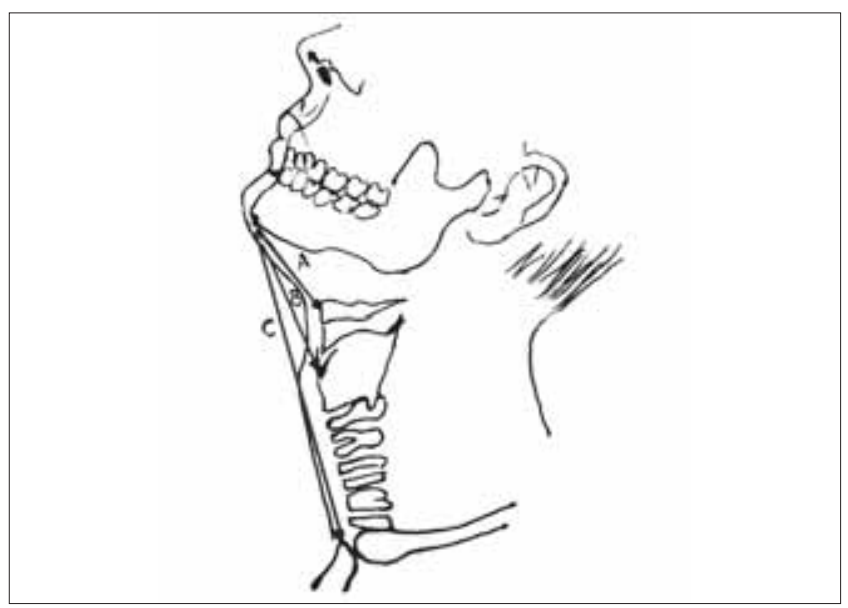

Figura 4. Algumas medidas realizadas no pré-operatório. Paciente com a cabeça em extenção. Distância hiomentual: da proeminência mentual ao osso hioide (A). Distância tireomentual: da proeminência mentual a proeminência da cartilagem tireoide (B). Distância esternomentual: da proeminência mentual ao bordo superior do manúbrio esternal (C).

O gap inter-incisivos foi medido com a boca aberta ao máximo, da ponta do incisivo superior ao inferior, em centímetros.

A posição neutra foi definida como o paciente sentado olhando reto na altura dos seus olhos, com a boca fechada. A posição com o pescoço em extensão foi obtida solicitando-se ao paciente para estender ao máximo seu pescoço.

A DHM, DTM e DEM foram medidas em centímetros, da proeminência mentual ao osso hioide, proeminência da cartilagem tireoide e bordo superior do manúbrio esternal, respectivamente (Figura 4). A MDHM foi medida 
QUADRO I. Escala proposta pelos autores: exposição das pregas vocais com o laringoscópio de suspensão.

\begin{tabular}{|c|c|c|}
\hline Classe & Exposicão Laríngea & Descrição da Exposição das Pregas Vocais \\
\hline Classe I & Ideal & $\begin{array}{l}\text { Visualização completa das pregas vocais, até a comissura } \\
\text { anterior }\end{array}$ \\
\hline Classe II & Inadequada & Visualização até terço médio das pregas vocais \\
\hline Classe III & Dificuldade de exposição laríngea propriamente dita & Visualização até terço posterior das pregas vocais \\
\hline Classe IV & Dificuldade de exposição laríngea propriamente dita & Visualização apenas da parede posterior da hipofaringe \\
\hline
\end{tabular}

em centímetros do ângulo da mandíbula até a proeminência mentual.

\section{Avaliação intra-operatória}

Durante a intubação endotraqueal, o anestesista avaliava a visibilidade laríngea com o laringoscópio, conforme a escala de Cormack- Lehane: classe 1 = toda a glote é visível; classe 2 = as cordas vocais são visíveis parcialmente ou apenas a comissura posterior; classe $3=$ somente a epiglote é visível e classe $4=$ a epiglote não é visível (1).

Com o laringoscópio de suspensão, no mínimo dois cirurgiões, diferentes dos que aplicaram o protocolo préoperatório, avaliavam a visualização laríngea. Classificavam conforme a escala proposta pelos autores (Quadro 1). Antes da utilização do suspensor e após a colocação do suspensor e, se necessário, da utilização de fita de esparadrapo e/ou compressão manual.

\section{Valores de referência}

Foram considerados como valores limites e avaliados estatisticamente como preditores de dificuldade de exposição laríngea: IMC $>25$, circunferência cervical $>40$ $\mathrm{cm}$, gap inter-incisivos $\leq 4 \mathrm{~cm}, \mathrm{DHM}<6,05 \mathrm{~cm}$, DHM/ DHMn $<1,2 \mathrm{~cm}$, DTM $<7,15 \mathrm{~cm}$, DEM $<13,9 \mathrm{~cm}$ e MDHM $\leq 9 \mathrm{~cm}(4,5)$.

Definimos as classes propostas pelos autores, após a colocação do suspensor de laringe, da seguinte maneira: classe $\mathrm{I}=$ exposição ideal da laringe, classe $\mathrm{II}=$ exposição inadequada e classes III e IV= dificuldade de exposição laríngea (Quadro 1). A classe I foi considerada como grupo controle e comparada com os demais.

\section{Análise estatística}

De acordo com a natureza dos dados analisados procedeu-se ao tratamento estatístico julgado adequado. Foram aplicados os testes: $t$ de Student para a diferença entre as médias de idade e IMC, entre os grupos I e II, da escala proposta pelos autores com o uso do suspensor de laringoscópio, observando a distribuição normal (gaussiana) das variáveis; de diferença de proporções antes e após a colocação de suspensor do laringoscópio para o grupo total; Qui-quadrado ou Fisher, quando uma das células com $\mathrm{n}<5$, para as demais variáveis entre as classes I e II proposta pelos autores com o uso do suspensor do laringoscópio. O nível de significância adotado foi $\mathrm{p}<0,05$.

\section{RESUltados}

Foram incluídos 57 pacientes no trabalho, sendo 30 pacientes do sexo feminino (52,63\%) e 27 do masculino (47,37\%). A idade variou de 14 a 66 anos, com média de 42 anos.

A indicação cirúrgica mais prevalente foi de pólipos, com 21 casos (36,84\%), seguido de 9 casos de papilomatose laríngea (15,79\%), 9 de cistos intra-cordais $(15,79 \%), 5$ de nódulos vocais $(8,77 \%), 4$ de lesões estruturais míninas (7,02\%), 4 de edema de Heinke (7,02\%), 2 leucoplasias (3,51\%), 2 granulomas pós-intubação (3,51\%) e 1 úlcera traumática $(1,75 \%)$.

Os pacientes foram classificados conforme a escala proposta pelos autores antes e após a colocação do suspensor do laringoscópio. Após a colocação do suspensor do laringoscópio, a maioria dos pacientes, 46 (80,70\%), apresentou exposição ideal das PPVV (classe I) e 11 pacientes obtiveram exposição inadequada da laringe (classe II). Estes dois grupos foram avaliados estatisticamente. Não obtivemos nenhum paciente com dificuldade de exposição laríngea (classes III e IV). Com o suspensor do laringoscópio um paciente da classe IV melhorou sua exposição laríngea para a classe II, 4 casos da classe III foram para a classe II, 2 evoluíram da classe III para a I, 6 se mantiveram na classe II e 13 da classe II obtiveram exposição ideal (classe I) (Tabela 1).

Comparando o grupo controle com o grupo de exposição inadequada da laringe, quanto aos fatores epidemiológicos: idade (Tabela 2$), \operatorname{sexo}(p=0,59)$ e indicações cirúrgicas, não houve diferença estatística. 
Tabela I. Análise estatística das proporções das classes propostas pelos autores antes e após a colocação do suspensor do laringoscópio.

\begin{tabular}{lccc}
\hline & \multicolumn{2}{c}{ ESCALA } & $p$ \\
& ANTES & APÓS & \\
\hline Classe I & 3 I (54,39\%) & $46(80,70 \%)^{*}$ & 0,04 \\
Classe II & $19(33,33 \%)$ & $1 \mid(19,30 \%) * *$ & 0,07 \\
Classe III & $6(10,53 \%)$ & 0 & - \\
Classe IV & I $(1,75 \%)$ & 0 & - \\
\hline TOTAL & 57 & 57 &
\end{tabular}

Nota: * grupo controle; ${ }^{* *}$ grupo exposição inadequada da laringe; p - nível de significância estatística.
Tabela 2. Análise estatística da média de idade entre o grupo controle e o grupo de exposição inadequada da laringe.

\begin{tabular}{lccccc}
\hline Grupos & \multicolumn{3}{c}{ Idade } & & $p$ \\
& min-max & Média & \pm & $d p$ & \\
\hline Controle & $14-66$ & 42,30 & \pm & 13,15 & $0,37 \mid 1$ \\
$\begin{array}{l}\text { Exposiçãa } \\
\text { inadequada }\end{array}$ & $22-60$ & 43,72 & \pm & 11,20 & \\
\hline
\end{tabular}

Nota: min-max - valores mínimo e máximo, dp - desviopadrão, p - nível de significância estatística

Tabela 3. Análise estatística dos parâmetros da anamnese e do exame físico como testes preditores de exposição inadequada das pregas vocais.

\begin{tabular}{lcccc}
\hline Parâmetros & Sensibilidade(\%) & Especificidade(\%) & VPP(\%) & VPN(\%) \\
\hline Roncos presentes & 55 & 52 & 21 & 83 \\
Alteração cervical presente & 9 & 89 & 17 & 80 \\
IMC $>25$ & 73 & 48 & 25 & 88 \\
Circunferência cervical $>40 \mathrm{~cm}$ & 18 & 61 & 10 & 76 \\
Ausência dentes & 45 & 50 & 18 & 79 \\
Mallampatialto & 9 & 77 & 8 & 78 \\
MLS alto & 55 & 65 & 27 & 86 \\
Gap inter-incisivos e" 4cm & 18 & 61 & 10 & 76 \\
Flexão pescoço limitada & 0 & 93 & 0 & 80 \\
Extensão pescoço limitada & 9 & 91 & 20 & 81 \\
Cormack Lehane e" 2 & 82 & 59 & 32 & 93 \\
DHM/DHMn $<1,2 \mathrm{~cm}$ & 18 & 91 & 33 & 82 \\
DHM $<6,05 \mathrm{~cm}$ & 100 & 48 & 31 & 0 \\
DTM $<7,15 \mathrm{~cm}$ & 45 & 80 & 36 & 82 \\
DEM $<13,9 \mathrm{~cm}$ & 9 & 98 & 50 & 82 \\
MDHM d" $9 \mathrm{~cm}$ & 9 & 98 & 50 &
\end{tabular}

Legenda: $\mathrm{VPP}=$ valor preditivo positivo; $\mathrm{VPN}=$ valor preditivo negativo; $\mathrm{IM}=$ índice de massa corporal; Índice Mallampati alto= classe 3 e 4; MLS alto= Teste mordida lábio classes 2 e 3; DHM/DHMn= relação distância hiomentual em extensão/neutra; DHM= distância hiomentual em extensão; DTM= distância tireomentual em extensão; DEM= distância esternomentual em extensão; MDHM= maior distância horizontal da mandíbula. Marcado em negrito e sublinhado os valores maiores que $70 \%$, valor da porcentagem escolhido aleatoriamente.

Os parâmetros da anamnese e do exame físico estudados foram avaliados como testes preditores de exposição laríngea inadequada (Tabela 3).

Dos parâmetros avaliados na anamnese, 28 apresentavam roncos noturnos e 6 alterações cervicais (Tabela 4). Destes, 4 tinham história de trauma cervical sem fratura, 1com torcicolos esporádicos e 1 com dor a rotação lateral do pescoço.

Dos dados avaliados no exame físico, o IMC no grupo total variou de 19 a 39,3; média de 26,45 (Tabela 5).
Um paciente havia sido submetido à cirurgia de correção de micrognatia, possuía MDHM de $10 \mathrm{~cm}$, apresentou visualização ideal da laringe.

As variáveis contínuas foram analisadas quanto a sua média, dp e valor de $\mathrm{r}$ (Tabela 6).

\section{DISCUSSÃO}

Os estudos prévios não são homogêneos quanto à visualização laríngea após a introdução do laringoscópio de 
Tabela 4. Análise estatística das ALTERAÇÕES na anamnese e exame físico entre o grupo controle e o grupo com exposição inadequada da laringe.

\begin{tabular}{|c|c|c|c|c|}
\hline & \multicolumn{2}{|c|}{ GRUPO } & \multirow{2}{*}{ TOTAL } & \multirow{2}{*}{$P$} \\
\hline & Controle & $\begin{array}{l}\text { Exposição } \\
\text { inadequada }\end{array}$ & & \\
\hline Roncos presentes & $22(48 \%)$ & $6(55 \%)$ & $28(49 \%)$ & 0,69 \\
\hline Alteração cervical presente & $5(\mid 1 \%)$ & | (9\%) & $6(11 \%)$ & 0,67 \\
\hline Ausência de dentes & $23(50 \%)$ & $5(45 \%)$ & $28(49 \%)$ & 0,78 \\
\hline Circunferência cervical >40 cm & $18(39 \%)$ & $2(18 \%)$ & $20(35 \%)$ & 0,17 \\
\hline Flexão reduzida pescoço & $3(7 \%)$ & $0(0 \%)$ & $3(5 \%)$ & 0,51 \\
\hline Extensão reduzida pescoço & 4 (9\%) & I ((9\%) & $5(9 \%)$ & 0,67 \\
\hline Índice Mallampatialto & II (24\%) & I (9\%) & $12(2 \mid \%)$ & 0,26 \\
\hline Gap inter-incisivos $\leq 4 \mathrm{~cm}$ & 18 (39\%) & $2(18 \%)$ & $20(35 \%)$ & 0,34 \\
\hline Teste mordida lábio superior alto & $16(35 \%)$ & $6(55 \%)$ & 22 (39\%) & 0,19 \\
\hline Índice de Cormack-Lehane $\geq 2$ & $19(4 \mid \%)$ & $9(82 \%)$ & $28(49 \%)$ & $0,04 *$ \\
\hline $\mathrm{DHM} / \mathrm{DHMn}<1,2 \mathrm{~cm}$ & 4 (9\%) & $2(18 \%)$ & $6(11 \%)$ & 0,71 \\
\hline $\mathrm{DHM}<6,05 \mathrm{~cm}$ & $24(52 \%)$ & II (I00\%) & $35(6 \mid \%)$ & $0,003 *$ \\
\hline $\mathrm{DTM}<7,15 \mathrm{~cm}$ & $9(20 \%)$ & $5(45 \%)$ & $14(25 \%)$ & 0,08 \\
\hline $\mathrm{DEM}<13,9 \mathrm{~cm}$ & | (2\%) & | (9\%) & $2(4 \%)$ & 0,35 \\
\hline $\mathrm{MDHM} \leq 9 \mathrm{~cm}$ & I (2\%) & I (9\%) & $2(4 \%)$ & 0,35 \\
\hline TOTAL & 46 & II & 57 & \\
\hline
\end{tabular}

Tabela 5. Análise estatística da média de imc entre o grupo controle e o grupo com exposição inadequada da laringe.

\begin{tabular}{|c|c|c|c|c|c|}
\hline \multirow[t]{2}{*}{ GRUPOS } & \multicolumn{4}{|c|}{ IMC } & \multirow{2}{*}{$p$} \\
\hline & Min-max & Média & \pm & Dp & \\
\hline $\begin{array}{l}\text { Controle } \\
\text { Exposição }\end{array}$ & $19-39,3$ & 26,37 & \pm & 4,58 & 0,35 \\
\hline inadequada & $22,2-32,9$ & 26,93 & \pm & 3,39 & \\
\hline
\end{tabular}

Nota: min-max - valores mínimo e máximo, dp - desviopadrão, p - nível de significância estatística.

suspensão. Alguns trabalhos dividem os pacientes em dois grupos: grupo de dificuldade de exposição laríngea, os pacientes com exposição limitada ao terço posterior ou menos das PPVV, e grupo controle, os demais pacientes $(5,6,7)$. Não avaliando os casos de exposição inadequada da laringe.

Outros estudos utilizam uma escala visual de 1 a 10 (8), considerada pelos próprios autores, como trabalhosa e subjetiva.

Há ainda trabalhos que consideram a escala de Cormark-Lehane. Em virtude do material utilizado, das manobras e dos objetivos do anestesista na intubação endotraqueal diferirem dos do cirurgião de laringe, a utilização da mesma escala, por ambos, não parece apropriada.
Tabela 6. Análise estatística da média das varíaveis contínuas do exame físico no grupo total.

\begin{tabular}{lcccc}
\hline & Mediana & Média & $\pm \mathrm{dp}$ & $\mathrm{R}$ \\
\hline IMC & 26 & 26,47 & 4,355 & 0,04 \\
Circunferência cervical & 38 & 38,61 & 4,366 & 0 \\
DHM & 6 & 6,21 & 1,235 & $-0,45$ \\
DTM & 8 & 8,35 & 1,316 & $-0,34$ \\
DEM & 17 & 17,29 & 2,218 & $-0,22$ \\
MDHM & 12 & 11,84 & 1,544 & $-0,09$ \\
\hline
\end{tabular}

Legenda: $\mathrm{IMC}=$ índice de massa corporal; $\mathrm{DHM}=$ distância hiomentual em extensão; DTM= distância tireomentual em extensão; DEM= distância esternomentual em extensão; MDHM= maior distância horizontal da mandíbula; $\mathrm{R}$ - coeficiente de correlação de Pearson

Percebe-se a inexistência de uma escala padrão de fácil aplicação e que supra as necessidades do cirurgião de laringe, somada a falta de padronização, dificultando a comparação entre estudos.

Os anestesistas consideram dificuldade de intubação endotraqueal as classes $3 \mathrm{e} 4 \mathrm{da}$ escala de Cormack-Lehane. Propomos considerar as classes III e IV da nova escala (Quadro 1) como de dificuldade de locação do laringoscópio e portanto, de dificuldade de exposição laríngea. Sugerimos também, considerar outras subdivisões: a classe II, como de exposição inadequada e a classe I, como exposição ideal. Pois, para o cirurgião de laringe, esta subdivisão pode afetar a realização e o resultado da cirurgia.

A nova escala, proposta pelos autores, (Quadro 1) parece ser apropriada e de fácil utilização, para avaliação 
da exposição laríngea durante a microcirurgia da laringe. Apropriada porque foca nas PPVV e considera a exposição da comissura anterior. De fácil utilização por utilizar apenas 4 classes intuitivas.

Diferindo dos estudos anteriores, não obtivemos nenhum caso de dificuldade de exposição laríngea propriamente dita. Encontramos apenas 11 casos (19,30\%) de exposição inadequada da laringe. Ressaltamos que em nosso estudo havia pacientes candidatos a difícil exposição. Como por exemplo, a média do IMC foi em torno de 26, ou seja, acima do valor considerado preditivo para dificuldade de exposição (Tabela 5). Ou ainda, 20 pacientes possuíam circunferência cervical acima do valor de corte, $40 \mathrm{~cm}$ (Tabela 4). Questionam-se fatores raciais, interferindo nos valores referências das variáveis, e o papel dos diferentes tipos de laringoscópios, afetando a comparação entre os diferentes estudos $(5,6)$. Talvez, uma limitação deste estudo, seja a utilização de apenas um tipo de laringoscópio pouco utilizado, o laringoscópio de Dan. Utilizado neste trabalho, por ser a preferência e rotina dos autores.

Como esperado, o suspensor do laringoscópio obteve melhora significativa na exposição das pregas vocais ( $\mathrm{p}=0,04$ para classe I). Vinte pacientes (35\%) melhoraram a exposição com seu uso (Tabela 1).

Neste estudo, o escore de exposição laríngea apresentou correlação significativa com o escore de CormackLehane utilizado pelos anestesistas. Isto, demonstra que os pacientes com difícil intubação endotraqueal são também candidatos a exposição laríngea inapropriada. Fato confirmado em outros estudos $(5,6,8)$.

Hsiung et al encontraram o sexo como o fator mais significativo em predizer dificuldade de exposição laríngea (6). Neste estudo, não houve diferença quanto ao sexo. Assim como para os demais fatores epidemiológicos analisados, idade e indicação cirúrgica. Os 2 grupos apresentaram idade, indicação cirúrgica e distribuição por sexo semelhantes (Tabela 2).

A avaliação da indicação cirúrgica, como fator preditivo de dificuldade de exposição laríngea, foi uma inovação deste estudo.

Vários parâmetros tiveram um índice de sensibilidade alto, como: IMC $>25$, índice de Cormarck-Lehane $\geq 2$, DHM. Alta especificidade: índice de Mallampati $\geq 2$, flexão limitada do pescoço, entre outros. Ou ainda, alto VPN, como: índice de Cormarck-Lehane $\geq 2$, DTM $<7,15 \mathrm{~cm}$, entre outros (Tabela 3). Porém, só atingimos significância estatística com a DHM $(\mathrm{p}=0,003)$ e índice de CormarckLehane $\geq 2(p=0,04)$ (Tabela 4).
Pesquisamos a presença de roncos noturnos, como um indicador de apneia obstrutiva do sono (SAOS), uma vez que pacientes com SOAS costumam ter dificuldade de intubação $(8,9)$. Entretanto a presença de roncos foi similar nos 2 grupos.

PINAR et al (5) notoram que as medidas do exame físico com o pescoço em extensão foram preditores mais fidedignos do que com a cabeça em posição neutra. Provavelmente, por serem mais próximas a utilizada durante a locação do laringoscópio. Portanto, só avaliamos as medidas em extensão.

PINAR et al associaram o IMC, a circunferência cervical e as medidas da DHM e DTM em extensão, com dificuldade de exposição laríngea (5). Avaliamos todos estes parâmetros. Entretanto, apenas a DHM $<6,05 \mathrm{~cm}$ teve correlação estatística com a exposição inadequada $(p=0,003)$, com alta sensibilidade.

\section{CONCLUSÃO}

Os únicos parâmetros que mostraram correlação significativa com a exposição inadequada da laringe foram a distância hiomentual com extensão da cabeça menor que $6,05 \mathrm{~cm}(\mathrm{p}=0,003)$ e índice de Cormarck-Lehane $\geq 2$ $(\mathrm{p}=0,04)$.

O uso do suspensor do laringoscópio melhorou a visualização laríngea de forma significativa $(p<0,04)$.

A escala proposta pelos autores parece adequada e de fácil aplicação para avaliação da exposição laríngea durante a microcirurgia da laringe. Com a visualização das PPVV subclassificada e padronizada, a comunicação entre otorrinolaringologistas deverá ser facilitada assim como estudos futuros.

\section{REFERÊNCIAS BIBLIOGRÁFICAS}

1. Cormack RS, Lehane J. Difficult tracheal intubation in obstetrics. Anaesthesia. 1984, 39:1105-1111.

2. Khan ZH, Kashfi A, Ebrahimkhani E. A comparison of the upper lip bite test (a simple new technique) with modified Mallampati classification in predicting difficulty in endotracheal intubation: a prospective blinded study. Anesth Analg. 2003, 96:595-599.

3. Hochman II, Zeitels SM, Heaton JT. Analysis of the forces and position required for direct laryngoscopic exposure of the anterior vocal folds. Ann Otol Rhinol Laryngol. 1999, 108:715-724. 
4. Merah NA, Wong DT, Ffoulkes-Crabbe DJ, Kushimo OT, Bode CO. Modified Mallampati test, thyromental distance and inter-incisor gap are the best predictors of difficult laryngoscopy in West Africans. Can J Anaesth. 2005, 52:291-296.

5. Pinar E, Calli C, Oncel S, Selek B, Tatar B. Preoperative clinical prediction of difficult laryngeal exposure in suspension laryngoscope. Eur Arch Otor. 2009, 266:699-703.

6. Hsiung MW, Pai L, Kang BH et al. Clinical predictors of difficult laryngeal exposure. Laryngoscope. 2004, 114:358363.

7. Kawaida M, Fukuda H, Kohno N. Video-assisted rigid endoscopic laryngosurgery: Application to cases with difficult laryngeal exposure. J. Voice. 2001, 15:305-312.

8. Hekiert AM, Mick R, Mirza N. Prediction of difficult laryngoscopy: does obesity play a role? Ann. Otol. Rhinol. Laryngol. 2007, 116(11):799-804.

9. Mallampati SR, Gatt SP, Gugino LD et al. A clinical sign to predict difficult tracheal intubation: a prospective study. Can. Anaesth. Soc. J. 1985, 32:429-434.

10. Hiremath AS, Hillman DR, James AL et al. Relationship between difficult tracheal intubation and obstructive sleep apnea. Br. J. Anaesth. 1998, 80:606-611. 